\title{
Global existence and blow-up of solutions for a nonlinear wave equation with memory
}

Fei Liang ${ }^{1,2}$ and Hongjun Gao ${ }^{1 *}$

\footnotetext{
* Correspondence: gaohj@njnu. edu.cn

${ }^{1}$ Jiangsu Provincial Key Laboratory for Numerical Simulation of Large Scale Complex Systems, School of Mathematical Science, Nanjing Normal University, Nanjing 210046, P.R. China

Full list of author information is available at the end of the article
}

\section{Abstract}

In this article, we consider the nonlinear viscoelastic equation

$$
u_{t t}-\Delta u+\int_{0}^{t} g(t-\tau) \Delta u(\tau) d \tau-\omega \Delta u_{t}+\mu u_{t}=|u|^{p-2} u
$$

with initial conditions and Dirichlet boundary conditions. We first prove a local existence theorem and show, for some appropriate assumption on $g$ and the initial data, that this solution is global with energy which decays exponentially under the potential well. Secondly, not only finite time blow-up for solutions starting in the unstable set is proved, but also under some appropriate assumptions on $g$ and the initial data, a blow-up result with positive initial energy is established. Finally, we also prove the boundedness of global solutions for strong $(\omega>0)$ damping case. 2000 MSC: 35L05; 35L15; 35L70.

Keywords: global existence, blow-up, wave equation, memory

\section{Introduction}

In this article we study the behavior of solutions for the following nonlinear viscoelastic equation

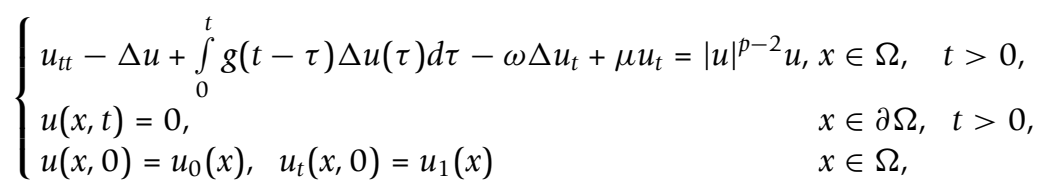

where $\Omega$ is a bounded domain in $\mathbb{R}^{n}$ with a smooth boundary $\partial \Omega, g$ is a positive function satisfying some conditions to be specified later, $\omega, \mu$ satisfy

$$
\omega \geq 0, \quad \mu>-\lambda \omega,
$$

$\lambda$ being the first eigenvalue of the operator $-\Delta$ under homogeneous Dirichlet boundary conditions, and

$$
2<p \leq\left\{\begin{array}{l}
\frac{2 n}{n-2}, \text { for } \omega>0, \\
\frac{2 n-2}{n-2}, \text { for } \omega=0,
\end{array} \text { if } n \geq 3,2<p<\infty \text { if } n=1,2\right.
$$

\section{Springer}


This problem has its origin in the mathematical description of viscoelastic materials. It is well known that viscoelastic materials exhibit natural damping, which is due to the special property of these materials to retain a memory of their past history. A general theory concerning problem (1.1) in the case $\omega=0$ and $\mu=0$ is available in literature (see [1-4]). The asymptotic behavior of the solutions to (1.1) has been studied in [5-8], we also refer to $[9,10]$ for the asymptotic decay of the solutions to problems analogous to (1.1). Among other known results about problem (1.1) with $\omega=0$ and $\mu=0$, we recall that in $[7,8]$, it is proved that the exponential decay of $g$ is a sufficient condition to the exponential decay of the solution $u$. In [5] it is also proved that, when $\omega=0$ and $\mu=0$, the exponential decay of $g$ is necessary for the exponential decay of $u$. When $\omega+\mu \neq 0$, Fabrizio and Polidoro [11] showed that the exponential decay of $g$ is a necessary condition for the exponential decay of $u$. The case of only having $\int_{0}^{t} g(t-\tau) \Delta u(\tau) d \tau$ may be very restrictive in many physical problems. Also, problem (1.1) is applied to the theory of the heat conduction with memory, see [12-16]. Therefore, the dynamics of (1.1) are of great importance and interest as they have wide applications in natural sciences.

This type of problem have been considered by many authors and several results concerning existence, nonexistence, and asymptotic behavior have been established. Cavalcanti et al. [17] studied the following equation:

$$
u_{t t}-\Delta u+\int_{0}^{t} g(t-\tau) \Delta u(\tau) d \tau+a(x) u_{t}+|u|^{\gamma} u=0, \quad \text { in } \Omega \times(0, \infty)
$$

for $a: \Omega \rightarrow \mathbb{R}^{+}$, a function, which may be null on a part of the domain $\Omega$. Under the conditions that $a(x) \geq a_{0}>0$ on $\Omega_{1} \subset \Omega$, with $\Omega_{1}$ satisfying some geometry restrictions and

$$
-\xi_{1} g(t) \leq g^{\prime}(t) \leq-\xi_{2} g(t), \quad t \geq 0,
$$

The authors established an exponential rate of decay. This latter result has been improved by Cavalcanti and Oquendo [18] and Berrimi and Messaoudi [19]. In their work, Cavalcanti and Oquendo [18] considered the situation where the internal dissipation acts on a part of $\Omega$ and the viscoelastic dissipation acts on the other part. They established both exponential and polynomial decay results under conditions on $g$ and its derivatives up to the third order, whereas Berrimi and Messaoudi [19] allowed the internal dissipation to be nonlinear. They also showed that the dissipation induced by the integral term is strong enough to stabilize the system and established an exponential decay for the solution energy provided that $g$ satisfies a relation of the form

$$
g^{\prime}(t) \leq-\xi g(t), \quad t \geq 0 .
$$

In [20], Berrimi and Messaoudi considered problem (1.1) for $\omega=\mu=0$. They established a local existence result and showed, for certain initial data and suitable conditions on $g$, that this solution is global with energy which decays exponentially or polynomially depending on the rate of the decay of the relaxation function $g$.

For nonexistence, we should mention that Messaoudi [21] looked into the equation

$$
u_{t t}-\Delta u+\int_{0}^{t} g(t-\tau) \Delta u(\tau) d \tau+|u|^{m-2} u=|u|^{p-2} u, \quad \text { in } \Omega \times(0, \infty)
$$


and proved, under appropriate relations between $p, m$ and $g$, a blow-up result. This work generalizes earlier ones by Georgiev and Todorova [22] and Messaoudi [23], in which a similar result has been established for the wave equation $(g \equiv 0)$. This result was later improved by Messaoudi [24], to certain solutions with positive initial energy. A similar result was also obtained by $\mathrm{Wu}$ [25] using a different method. For the problem (1.4) in $\mathbb{R}^{n}$ and with $m=2$, Kafini and Messaoudi [26] showed, for suitable conditions on $g$ and initial data, that solutions with negative energy blow up in finite time. More recently, Wang [27] has investigated a sufficient condition of the initial data with arbitrarily positive initial energy such that the corresponding solution of Equation (1.4) with $m=2$ blows up in finite time. This result improved the blow-up results in $[21,24]$.

In this article, we first consider (1.1) and establish a local existence result. In addition, using the ideas of the "potential well" theory introduced by Payne and Sattinger [28], we show that for some appropriate assumption on $g$ (but without exponential decay property) and the initial data, that this solution is global with energy which decays exponentially under the potential well. Secondly, not only finite time blow up for solutions starting in the unstable set is proved, but also under some appropriate assumptions on $g$ and the initial data, a blow-up result with positive initial energy is established. Finally, we also prove the boundedness of global solutions $u(t)$ to problem (1.1) for strong $(\omega>0)$ damping, namely, $u \in L^{\infty}\left(\mathbb{R}^{+} ; H_{0}^{1}(\Omega)\right) \cap W^{1, \infty}\left(\mathbb{R}^{+} ; L^{2}(\Omega)\right)$.

This article is organized as follows. In Section 2 we introduce some notation and prepare some material. Section 3 is devoted to global existence for solutions under the potential well and the decay result. In Section 4 we will show that there are solutions of (1.1) with positive initial energy or with arbitrary positive initial energy that blow up in finite time. The last Section we will prove the boundedness of global solutions $u(t)$ to problem (1.1) for strong $(\omega>0)$ damping.

\section{Preliminaries}

We denote by $\|\cdot\|_{q}$ the $L^{q}(\Omega)$ norm for $1 \leq q \leq \infty$ and by $\|\nabla \cdot\|_{2}$ the Dirichlet norm in $H_{0}^{1}(\Omega)$. Moreover, for later use we denote by $\langle\cdot, \cdot\rangle$ the duality pairing between $H^{-1}(\Omega)$ and $H_{0}^{1}(\Omega)$. When $\omega>0$ (resp. $\omega=0$ ) for $v, w \in H_{0}^{1}(\Omega)$ (resp. for all $v, w \in L^{2}(\Omega)$ ), we put

$$
(v, w)_{*}=\omega \int_{\Omega} \nabla v \cdot \nabla w+\mu \int_{\Omega} v w, \quad\|v\|_{*}=(v, v)_{*}^{1 / 2}
$$

by (1.2), $\|\cdot\|_{*}$ is an equivalent norm over $H_{0}^{1}(\Omega)\left(\right.$ resp. $\left.L^{2}(\Omega)\right)$.

Let $a>0$. Define $J_{a}, I_{a}: H_{0}^{1}(\Omega) \rightarrow \mathbb{R}$ by

$$
J_{a}=\frac{a}{2}\|\nabla u\|_{2}^{2}-\frac{1}{p}\|u\|_{p}^{p}, \quad I_{a}=a\|\nabla u\|_{2}^{2}-\|u\|_{p}^{p} .
$$

In this case, the "potential depth" is defined as

$$
d_{a}=\inf _{w \in H_{0}^{1}(\Omega) \backslash\{0\}} \max _{\lambda \geq 0} J_{a}(\lambda u) .
$$


It is easy to see that the "potential well" is positive, see $[28,29]$ for details. Next, we define stable and unstable sets respectively:

$$
\begin{gathered}
W_{a}=\left\{u \in H_{0}^{1}(\Omega) \mid I_{a}(u)>0, \quad J_{a}(u)<d_{a}\right\} \cup\{0\}, \\
V_{a}=\left\{u \in H_{0}^{1}(\Omega) \mid I_{a}(u)<0, \quad J_{a}(u)<d_{a}\right\} .
\end{gathered}
$$

Finally, we consider the energy functional $E(t)=E\left(u(t), u_{t}(t)\right)$ defined by

$$
E(t)=\frac{1}{2}\left\|u_{t}(t)\right\|_{2}^{2}+\frac{1}{2}\left(1-\int_{0}^{t} g(s) d s\right)\|\nabla u(t)\|_{2}^{2}+\frac{1}{2}(g \circ \nabla u)(t)-\frac{1}{p}\|u\|_{p}^{p},
$$

where

$$
(g \circ v)(t)=\int_{0}^{t} g(t-\tau)\|v(t)-v(\tau)\|_{2}^{2} d \tau .
$$

For the relaxation function $g(t)$ we assume

(G1) $g \in C^{1}[0, \infty)$ is a non-negative and non-increasing function satisfying

$$
\begin{aligned}
& 1-\int_{0}^{\infty} g(s) d s=1-\kappa=l>0 . \\
& \text { (G2) } \int_{0}^{\infty} g(s) d s<\frac{p / 2-1}{p / 2-1+1 /(2 p)} .
\end{aligned}
$$

Remark 2.1. Condition (1.3) is needed to establish the local existence result. In fact under this condition, the nonlinearity is Lipschitz from $H^{1}(\Omega)$ to $L^{2}(\Omega)$. Condition (G1) is necessary to guarantee the hyperbolicity and well-posedness of problem (1.1).

\section{Global existence and exponential energy decay}

In this section we study the global existence of solutions for problem (1.1). For this purpose, we first consider a related linear problem. Then, we use the well-known contraction mapping theorem to prove the existence of solutions to the nonlinear problem. Throughout the section, we restrict ourselves to the case $\omega>0, \mu \neq 0$ and $n \geq 3$, the other cases being similar (and simpler).

For a given $T>0$, we consider the space $\mathcal{H}=C\left([0, T] ; H_{0}^{1}(\Omega)\right) \cap C^{1}\left([0, T] ; L^{2}(\Omega)\right)$ equipped with the norm

$$
\|u\|_{\mathcal{H}}^{2}=\max _{0 \leq t \leq T}\left(l\|\nabla u(t)\|_{2}^{2}+\left\|u_{t}(t)\right\|_{2}^{2}\right) .
$$

Lemma 3.1. Assume (G1), (1.2) and (1.3) hold. For every $T>0$, every $u \in \mathcal{H}$ and every initial data $\left(u_{0}, u_{1}\right) \in H_{0}^{1}(\Omega) \times L^{2}(\Omega)$ there exists a unique

$$
v \in \mathcal{H} \cap C^{2}\left([0, T] ; H^{-1}(\Omega)\right) \quad \text { such that } v_{t} \in L^{2}\left([0, T] ; H_{0}^{1}(\Omega)\right),
$$

which solves the linear problem

$$
\begin{cases}v_{t t}-\Delta v+\int_{0}^{t} g(t-\tau) \Delta v(\tau) d \tau-\omega \Delta v_{t}+\mu v_{t}=|u|^{p-2} u, & (x, t) \in \Omega \times[0, T] \\ v(x, t)=0, & (x, t) \in \partial \Omega \times[0, T] \\ v(x, 0)=u_{0}(x), \quad v_{t}(x, 0)=u_{1}(x) & x \in \Omega .\end{cases}
$$


Proof. The proof follows from a directly application of the Galerkin method as in $[22,30]$, thus we omit it here.

Theorem 3.2. Assume (G1), (1.2) and (1.3) hold. For any initial data $\left(u_{0}, u_{1}\right) \in H_{0}^{1}(\Omega) \times L^{2}(\Omega)$, there exists a real number $T_{m}>0$ such that problem (1.1) has a unique local weak solution

$$
\begin{aligned}
& u \in \mathcal{H} \cap C^{2}\left(\left[0, T_{m}\right) ; H^{-1}(\Omega)\right) \quad \text { such that } u_{t} \in L^{2}\left([0, T] ; H_{0}^{1}(\Omega)\right) . \\
& \text { If } T_{m}<\infty \text {, then } \\
& \lim _{t \rightarrow T_{m}}\left(l\|\nabla u(t)\|_{2}^{2}+\left\|u_{t}(t)\right\|_{2}^{2}=\infty .\right)
\end{aligned}
$$

Proof. Taking $\left(u_{0}, u_{1}\right) \in H_{0}^{1}(\Omega) \times L^{2}(\Omega)$ and letting $R=2\left(\left\|\nabla u_{0}\right\|_{2}^{2}+\left\|u_{1}\right\|_{2}^{2}\right)$. For any $T>0$, we consider

$$
\mathcal{F}=\left\{u \in \mathcal{H}: u(0)=u_{0}, u_{t}(0)=u_{1} \text { and }\|u\|_{\mathcal{H}} \leq R\right\} .
$$

By Lemma 3.1, for any $u \in \mathcal{F}$ we may define $v=\Phi(u)$, being the unique solution to problem (3.1). We claim that, for a suitable $T>0, \Phi$ is a contractive map from $\mathcal{F}$ into itself. Given $u \in \mathcal{F}$, multiplying (3.1) by $v_{t}$ and integrating over $[0, t] \subset[0, T]$, we have

$$
\begin{aligned}
& \left\|v_{t}(t)\right\|_{2}^{2}+\left(1-\int_{0}^{t} g(s) d s\right)\|\nabla v(t)\|_{2}^{2}+(g \circ \nabla v)(t)+2 \int_{0}^{t}\left\|v_{t}(\tau)\right\|_{*}^{2} d \tau \\
& =\int_{0}^{t}\left(g^{\prime} \circ \nabla v\right) d \tau-\int_{0}^{t} g(\tau)\|\nabla v(\tau)\|_{2}^{2} d \tau+\left\|\nabla u_{0}\right\|_{2}^{2}+\left\|u_{1}\right\|_{2}^{2}+2 \int_{0}^{t} \int_{\Omega}|u(\tau)|^{p-2} u(\tau) v_{t}(\tau) d x d \tau \\
& \leq\left\|\nabla u_{0}\right\|_{2}^{2}+\left\|u_{1}\right\|_{2}^{2}+2 \int_{0}^{t} \int_{\Omega}|u(\tau)|^{p-2} u(\tau) v_{t}(\tau) d x d \tau,
\end{aligned}
$$

here taking into account the condition (G1). For the last term, using Hölder, Sobolev, and Young inequalities, we have

$$
\begin{aligned}
& 2 \int_{0}^{t} \int_{\Omega}|u(\tau)|^{p-2} u(\tau) v_{t}(\tau) d x d \tau \leq C \int_{0}^{T}\|u(\tau)\|_{2 *}^{p-1}\left\|v_{t}(\tau)\right\|_{2 *} d \tau \\
& \leq C_{1} \int_{0}^{T}\|u(\tau)\|_{*}^{p-1}\left\|v_{t}(\tau)\right\|_{*} d \tau \leq C_{2} T R^{2(p-1)}+2 \int_{0}^{T}\left\|v_{t}(\tau)\right\|_{*}^{2} d \tau,
\end{aligned}
$$

where $2^{*}=2 n /(n-2)$. Combining (3.3) with (3.4) and taking the maximum over [0, T], we get

$$
\|v\|_{\mathcal{H}}^{2} \leq \frac{1}{2} R^{2}+C_{2} T R^{2(p-1)} .
$$

Choosing $T$ sufficiently small such that $C_{2} T R^{2(p-1)} \leq R^{2} / 2$, we get $\|v\|_{\mathcal{H}} \leq R$, which shows that $\Phi$ maps $\mathcal{F}$ into itself.

Next, we verify that $\Phi$ is a contraction. Taking $w_{1}$ and $w_{2}$ in $\mathcal{F}$, subtracting the two equations (3.1) for $v_{1}=\Phi\left(w_{1}\right)$ and $v_{2}=\Phi\left(w_{2}\right)$ and setting $w=v_{1^{-}} v_{2}$, then we have for all $\varphi \in H_{0}^{1}(\Omega)$ and a.e. $t \in[0, T]$ 


$$
\begin{aligned}
& \left\langle w_{t t}, \varphi\right\rangle+\int_{\Omega} \nabla w(t) \cdot \nabla \varphi d x+\int_{\Omega} \int_{0}^{t} g(t-\tau) \Delta w(\tau) d \tau \varphi d x+\int_{\Omega} \nabla w_{t}(t) \cdot \nabla \varphi d x+\mu \int_{\Omega} w_{t}(t) \varphi d x \\
& =\int_{\Omega}\left(\left|w_{1}(t)\right|^{p-2} w_{1}(t)-\left|w_{2}(t)\right|^{p-2} w_{2}(t)\right) \varphi d x .
\end{aligned}
$$

By taking $\phi=w_{t}$ in (3.5) and arguing as above, we obtain

$$
\left\|\Phi\left(w_{1}\right)-\Phi\left(w_{2}\right)\right\|_{\mathcal{H}}^{2}=\|w\|_{\mathcal{H}}^{2} \leq C_{3} R^{2 p-4} T\left\|w_{1}-w_{2}\right\|_{\mathcal{H}}^{2} \leq \varepsilon\left\|w_{1}-w_{2}\right\|_{\mathcal{H}}^{2}
$$

for some $\varepsilon<1$ provided $T$ is sufficiently small. This proves the claim. By the contraction mapping principle, there exists a unique (weak) solution to (1.1) defined on [0, $\left.T_{m}\right)$.

By the construction above, we observe that the local existence time of $u$ merely depends (through $R$ ) on the norms of the initial data. Therefore, as long as $\|u(t)\|_{\mathcal{H}}$ remains bounded, the solution may be continued, see also [[31], p. 158], for a similar argument. Hence, if $T_{m}<\infty$, we have

$$
\lim _{t \rightarrow T_{m}}\left(l\|\nabla u(t)\|_{2}^{2}+\left\|u_{t}(t)\right\|_{2}^{2}\right)=\lim _{t \rightarrow T_{m}}\|u(t)\|_{\mathcal{H}}=\infty
$$

Before we state and prove our global existence result, we need the following lemmas.

Lemma 3.3. [24, Lemma 2.1] Assume (G1), (1.2) and (1.3) hold. Let $u(t)$ be a solution of (1.1). Then $E(t)$ is nonincreasing, that is

$$
E^{\prime}(t)=-\left\|u_{t}(t)\right\|_{*}^{2}+\frac{1}{2}\left(g^{\prime} \circ \nabla u\right)(t)-\frac{1}{2} g(t)\|\nabla u(t)\|_{2}^{2} \leq 0 .
$$

Moreover, the following energy inequality holds:

$$
E(t)+\int_{s}^{t}\left\|u_{t}(\tau)\right\|_{*}^{2} d \tau \leq E(s), \quad \text { for } 0 \leq s \leq t<T_{m}
$$

Lemma 3.4. Assume (G1), (1.2) and (1.3) hold, and $0<a \leq l$. Let $u(x, t)$ be a local solution of problem (1.1) with initial data $\left(u_{0}, u_{1}\right) \in H_{0}^{1}(\Omega) \times L^{2}(\Omega)$. Then the following assertions hold.

(1) If there exists a number $t_{0} \in\left[0, T_{m}\right)$ such that $u\left(\cdot, t_{0}\right) \in W_{a}$ and $E\left(t_{0}\right)<d_{a}$, then $u(\cdot$, $t) \in W_{a}$ and $E(t)<d_{a}$ for all $t \in\left[t_{0}, T_{m}\right)$.

(2) If there exists a number $t_{0} \in\left[0, T_{m}\right)$ such that $u\left(\cdot, t_{0}\right) \in V_{a}$ and $E\left(t_{0}\right)<d_{a}$, then $u(\cdot$, $t) \in V_{a}$ and $E(t)<d_{a}$ for all $t \in\left[t_{0}, T_{m}\right)$.

Proof. The proof is almost the same that of Tsutsumi [32].

The following integral inequality plays an important role in our proof of the energy decay of the solutions to problem (1.1).

Lemma 3.5. [33] Assume that the function $\phi: \mathbb{R}^{+} \cup\{0\} \rightarrow \mathbb{R}^{+} \cup\{0\}$ is a non-increasing function and that there exists a constant $c>0$ such that

$$
\int_{t}^{\infty} \varphi(s) d s \leq c \varphi(t)
$$


for every $t \in[0, \infty)$. Then

$$
\varphi(t) \leq \varphi(0) \exp (1-t / c)
$$

for every $t \geq c$.

Theorem 3.6. Assume (G1), (1.2) and (1.3) hold, and $0<a \leq l$. Let $u(x, t)$ be a local solution of problem (1.1) with initial data $\left(u_{0}, u_{1}\right) \in H_{0}^{1}(\Omega) \times L^{2}(\Omega)$. In addition assume that $u(0) \in W_{a}$ and $E(0)<d_{a}$, then the corresponding solution to (1.1) globally exists, i.e., $T_{m}=\infty$. Moreover, if $d_{a}<\theta$ and $\sigma=1-l>0$ is small sufficiently such that

$$
1-C^{p}(\Omega)\left(\frac{2 p E(0)}{p-2}\right)^{\frac{p-2}{2}} \frac{-p}{2}-\frac{5 p(1-l)}{2(p-2) l}>0
$$

where $\theta=(p-2) /(2 p)^{p /(p-2)} C^{-2 p /(p-2)}(\Omega)$ and $C(\Omega)$ is the optimal constant of Sobolev imbedding $H_{0}^{1}(\Omega) \hookrightarrow L^{p}(\Omega)$, then the energy decay is

$$
E(t) \leq E(0) \exp \left(1-C^{-1} t\right)
$$

for every $t \in[0, \infty)$, where $C$ is some positive constant.

Proof. We only consider the case $\omega>0$ and $\mu>-\lambda \omega$. In order to get $T_{m}=\infty$, by Theorem 3.2, it suffices to show that

$$
l\|\nabla u(t)\|_{2}^{2}+\left\|u_{t}(t)\right\|_{2}^{2}
$$

is bounded independently of $t$. Since $u(0) \in W_{a}$ and $E(0)<d_{a}$, it follows from Lemma 3.4 that

$$
u(t, \cdot) \in W_{a}, \quad E(t)<d_{a} \quad \text { for }\left[0, T_{m}\right) .
$$

On the other hand, since $u(t, \cdot) \in W_{a}$ means

$$
J_{a}(u) \geq \frac{(p-2) a}{2 p}\|\nabla u(t)\|_{2}^{2} \text { for }\left[0, T_{m}\right) .
$$

So, it follows from (3.8) and Lemma 3.3 with $s=0$ that

$$
\begin{aligned}
\frac{(p-2) a}{2 p} l\|\nabla u(t)\|_{2}^{2}+\frac{1}{2}\left\|u_{t}(t)\right\|_{2}^{2} & \leq J_{a}(u)+\frac{1}{2}\left\|u_{t}(t)\right\|_{2}^{2} \\
& \leq E(t)+\int_{0}^{t}\left\|u_{t}(\tau)\right\|_{*}^{2} d \tau \leq E(0)<d_{a} \text { for }\left[0, T_{m}\right),
\end{aligned}
$$

which implies

$$
l\|\nabla u(t)\|_{2}^{2}+\left\|u_{t}(t)\right\|_{2}^{2} \leq C d_{a}
$$

where $C$ is a positive constant depending only on $l$ and $p$.

From Lemma 3.3 we have

$$
E(0) \geq E(t) \geq \frac{1}{2}\left(1-\int_{0}^{t} g(s) d s\right)\|\nabla u(t)\|_{2}^{2}-\frac{1}{p}\|u\|_{p}^{p} \geq \frac{l}{2}\|\nabla u(t)\|_{2}^{2}-\frac{1}{p}\|u\|_{p}^{p},
$$


which together with $u(t, \cdot) \in W_{a}$ yields

$$
\|\nabla u(t)\|_{2}^{2} \leq \frac{2 p}{(p-2) l} E(t) \leq \frac{2 p}{(p-2) l} E(0) .
$$

In addition,

$$
\begin{aligned}
\left(1-\frac{2}{p}\right)\|u(t)\|_{p}^{p} & \leq\left(1-\frac{2}{p}\right) C^{p}(\Omega)\|\nabla u(t)\|_{2}^{p} \\
& \leq \frac{2}{l} C^{p}(\Omega)\left(\frac{2 p}{(p-2) l} E(0)\right)^{\frac{p-2}{2}} E(t) \leq 2(1-\varepsilon) E(t)
\end{aligned}
$$

where

$$
\varepsilon=1-C^{p}(\Omega)\left(\frac{2 p}{(p-2)} E(0)\right)^{\frac{p-2}{2}} \frac{-p}{2} .
$$

Note that $E(0)<\theta$, we see that $\epsilon>0$.

Multiplying (1.1) by $u(t)$ and integrating over $\Omega \times\left[t_{1}, t_{2}\right]\left(0 \leq t_{1} \leq t_{2}\right)$, we get

$$
\begin{aligned}
0= & \int_{\Omega} \int_{t_{1}}^{t_{2}} u\left(u_{t t}-\Delta u+\int_{0}^{t} g(t-\tau) \Delta u(\tau) d \tau-\omega \Delta u_{t}+\mu u_{t}-|u|^{p-2} u\right) d t d x \\
= & {\left[\int_{\Omega} u(t) u_{t}(t) d x\right]_{t_{1}}^{t_{2}}-\int_{t_{1}}^{t_{2}}\left\|u_{t}(t)\right\|_{2}^{2} d t+\int_{t_{1}}^{t_{2}}\|\nabla u(t)\|_{2}^{2} d t-\int_{t_{1}}^{t_{2}}\|u(t)\|_{p}^{p} d t } \\
& +\int_{t_{1}}^{t_{2}}\left(u(t), u_{t}(t)\right)_{*} d t+\int_{t_{1}}^{t_{2}} \int_{\Omega}^{t} \int_{0}^{t} g(t-\tau) \Delta u(\tau) u(t) d \tau d x d t \\
= & {\left[\int_{\Omega}^{t_{2}} u(t) u_{t}(t) d x\right]_{t_{1}}^{t_{2}}-2 \int_{t_{1}}^{t_{2}}\left\|u_{t}(t)\right\|_{2}^{2} d t+2 \int_{t_{1}}^{t_{2}} E(t) d t+\left(\frac{2}{p}-1\right) \int_{t_{1}}^{t_{2}}\|u(t)\|_{p}^{p} d t } \\
& -\int_{t_{1}}^{t_{2}}(g \circ \nabla u)(t) d t+\int_{t_{1}}^{t_{1}} \int_{0}^{t_{2}} g(\tau) d \tau\|\nabla u(t)\|_{2}^{2} d t+\int_{t_{1}}^{t_{2}}\left(u(t), u_{t}(t)\right)_{*} d t \\
& +\int_{t_{1}}^{t_{2}} \int_{\Omega} \int_{0}^{t} g(t-\tau) \Delta u(\tau) u(t) d \tau d x d t .
\end{aligned}
$$

For the last term in (3.11), one has

$$
\begin{aligned}
& -2 \int_{\Omega} \int_{0}^{t} g(t-\tau) \Delta u(\tau) u(\tau) d \tau d x=2 \int_{\Omega} \int_{0}^{t} g(t-\tau) \nabla u(\tau) \nabla u(t) d \tau d x \\
& =\int_{0}^{t} g(t-\tau)\left(\|\nabla u(t)\|_{2}^{2}+\|\nabla u(\tau)\|_{2}^{2}\right) d \tau-\int_{0}^{t} g(t-\tau)\left(\|\nabla u(t)-\nabla u(t)\|_{2}^{2}\right) d \tau .
\end{aligned}
$$


Combining (3.11) and (3.12), we have

$$
\begin{aligned}
& 2 \int_{t_{1}}^{t_{2}} E(t) d t+\left(\frac{2}{p}-1\right) \int_{t_{1}}^{t_{2}}\|u(t)\|_{p}^{p} d t \\
= & -\left[\int_{\Omega}^{t_{2}} u(t) u_{t}(t) d x\right]_{t_{1}}^{t_{2}}+2 \int_{t_{1}}^{t_{2}}\left\|u_{t}(t)\right\|_{2}^{2} d t+\frac{1}{2} \int_{t_{1}}^{t_{2}}(g \circ \nabla u)(t) d t \\
& -\frac{1}{2} \int_{t_{1}}^{t_{2}} \int_{0}^{t} g(\tau) d \tau\|\nabla u(t)\|_{2}^{2} d t+\frac{1}{2} \int_{t_{1}}^{t_{2}} \int_{0}^{t} g(t-\tau)\|\nabla u(\tau)\|_{2}^{2} d \tau d t-\int_{t_{1}}^{t_{2}}\left(u(t), u_{t}(t)\right)_{*} d t \\
\leq & -\left[\int_{\Omega} u(t) u_{t}(t) d x\right]_{t_{1}}^{t_{2}}+2 \int_{t_{1}}^{t_{2}}\left\|u_{t}(t)\right\|_{2}^{2} d t+\frac{1}{2} \int_{t_{1}}^{t_{2}}(g \circ \nabla u)(t) d t \\
& +\frac{1}{2} \int_{t_{1}}^{t_{2}} \int_{0}^{t} g(t-\tau)\|\nabla u(\tau)\|_{2}^{2} d \tau d t-\int_{t_{1}}^{t_{2}}\left(u(t), u_{t}(t)\right)_{*} d t
\end{aligned}
$$

where the last inequality comes from (G1). For the left-hand side of the (3.13), by (3.10) we obtain

$$
2 \int_{t_{1}}^{t_{2}} E(t) d t+\left(\frac{2}{p}-1\right) \int_{t_{1}}^{t_{2}}\|u(t)\|_{p}^{p} d t \geq 2 \varepsilon \int_{t_{1}}^{t_{2}} E(t) d t
$$

We next estimate every term of the right-hand side of the (3.13). Firstly, by Hölder inequality and Poincaré inequality

$$
\int_{\Omega}\left|u(t) u_{t}(t)\right| d x \leq \frac{1}{2}\|u(t)\|_{2}^{2}+\frac{1}{2}\left\|u_{t}(t)\right\|_{2}^{2} \leq \frac{\lambda}{2}\|\nabla u(t)\|_{2}^{2}+E(t) .
$$

Using (3.9) we see that

$$
\int_{\Omega}\left|u(t) u_{t}(t)\right| d x \leq c_{1} E(t)
$$

where $c_{1}$ is a constant independent on $u$, from which follows that

$$
\left[\int_{\Omega} u(t) u_{t}(t) d x\right]_{t_{1}}^{t_{2}} \leq 2 c_{1} E\left(t_{1}\right) .
$$

Since $u(t, \cdot) \in W_{a}$, we have $0<I_{a}(u) \leq E(t)$. Thus, from (3.7), we deduce that

$$
\int_{t_{1}}^{t_{2}}\left\|u_{t}(t)\right\|_{*}^{2} \leq E\left(t_{1}\right)
$$

which implies

$$
\int_{t_{1}}^{t_{2}}\left\|\nabla u_{t}(t)\right\|_{2}^{2} \leq c_{2} E\left(t_{1}\right)
$$


Hence, by Poincaré inequality we get

$$
2 \int_{t_{1}}^{t_{2}}\left\|u_{t}(t)\right\|_{2}^{2} d t \leq 2 c_{3} E\left(t_{1}\right)
$$

where $c_{3}$ is a constant independent on $u$. In addition, using Young's inequality for convolution $\left\|f^{*} g\right\|_{q} \leq\|f\|_{r}\|g\|_{s}$ with $1 / q=1 / r+1 / s-1$ and $1 \leq q, r, s \leq \infty$, noting that if $q=1$, then $r=1$ and $s=1$, we have

$$
\int_{t_{1}}^{t_{2}} \int_{0}^{t} g(t-\tau)\|\nabla u(\tau)\|_{2}^{2} d \tau d t \leq \int_{t_{1}}^{t_{2}} g(t) d t \int_{t_{1}}^{t_{2}}\|\nabla u(t)\|_{2}^{2} d t \leq(1-l) \int_{t_{1}}^{t_{2}}\|\nabla u(t)\|_{2}^{2} d t .
$$

Further, by (3.9) we then have

$$
\int_{t_{1}}^{t_{2}} \int_{0}^{t} g(t-\tau)\|\nabla u(\tau)\|_{2}^{2} d \tau d t \leq \frac{2 p(1-l)}{(p-2) l} \int_{t_{1}}^{t_{2}} E(t) d t
$$

and

$$
\int_{t_{1}}^{t_{2}} \int_{0}^{t} g(t-\tau)\|\nabla u(\tau)\|_{2}^{2} d \tau d t \leq \frac{2 p(1-l)}{(p-2) l} \int_{t_{1}}^{t_{2}} E(t) d t
$$

Combining (3.17) and (3.18), we get

$$
\frac{1}{2} \int_{t_{1}}^{t_{2}}(g \circ \nabla u)(t) d t \int_{t_{1}}^{t_{2}} \int_{0}^{t} g(t-\tau)\left(\|\nabla u(t)\|_{2}^{2}+\|\nabla u(\tau)\|_{2}^{2}\right) d \tau d t \leq \frac{4 p(1-l)}{(p-2) l} \int_{t_{1}}^{t_{2}} E(t) d t .
$$

By Poincaré inequality and (3.9), we also have the following estimate

$$
\begin{aligned}
& -2 \int_{t_{1}}^{t_{2}}\left(u(t), u_{t}(t)\right)_{*} d t=-\int_{t_{1}}^{t_{2}} \frac{d}{d t}\|u(t)\|_{*}^{2}=\left\|u\left(t_{1}\right)\right\|_{*}^{2}-\left\|u\left(t_{2}\right)\right\|_{*}^{2} \\
\leq & 2 \frac{\lambda \omega+\mu}{\lambda}\left\|\nabla u\left(t_{1}\right)\right\|_{2}^{2} \leq \frac{4 p(\lambda \omega+\mu)}{\lambda(p-1) l} E\left(t_{1}\right) \leq c_{4} E\left(t_{1}\right),
\end{aligned}
$$

where $c_{4}$ is a constant independent on $u$.

Combining (3.13)-(3.20), we obtain

$$
2 \varepsilon \int_{t_{1}}^{t_{2}} E(t) d t \leq 2 C E\left(t_{1}\right)+\frac{5 p(1-l)}{(p-2) l} \int_{t_{1}}^{t_{2}} E(t) d t
$$

where $C$ is a constant independent on $u$, that is

$$
\left(1-C^{p}(\Omega)\left(\frac{2 p E(0)}{p-2}\right)^{\frac{p-2}{2}} l^{\frac{-p}{2}}-\frac{5 p(1-l)}{2(p-2) l}\right) \int_{t_{1}}^{t_{2}} E(t) d t \leq C E\left(t_{1}\right) .
$$

Denote

$$
a=1-C^{p}(\Omega)\left(\frac{2 p E(0)}{p-2}\right)^{\frac{p-2}{2}} l^{\frac{-p}{2}}-\frac{5 p(1-l)}{2(p-1) l} .
$$


We rewrite (3.21)

$$
a \int_{t}^{\infty} E(\tau) d \tau \leq C E(t)
$$

for every $t \in[0, \infty)$.

Since $a>0$ when $\sigma=1-l>0$ small sufficiently by Lemma 3.5 , we obtain the following energy decay for problem (1.1) as

$$
E(t)<E(0) \exp \left(1-a C^{-1} t\right)
$$

for every $t \geq C a^{-1}$.

Remark 3.1. For the definition of $d_{a}$ and Sobolev imbedding inequality, we have

$$
d_{a} \leq \frac{p-2}{2 p} a^{\frac{p}{p-2}}\left(\frac{\|\nabla u\|_{2}}{\|u\|_{p}}\right)^{\frac{2 p}{p-2}}
$$

and

$$
\|u\|_{p} \leq C\|\nabla u\|_{2} .
$$

Since $L^{p}(\Omega) \hookrightarrow H_{0}^{1}(\Omega)$ is compact, the best constants and the best function $v(x)$ in the above Sobolev imbedding inequality can be attained. For example, $n=1, p=\infty, \Omega$ $=(c, d) \subset \mathbb{R}$, the best $C$ and the best function $v(x)$ are attained, see [34]. In this case, $d_{a} \leq \frac{p-2}{2 p} a^{\frac{p}{p-2}} C^{\frac{-2 p}{p-2}}<\theta$. Then, we can take the initial data $u_{0}=v(x)$ which yields the set of the initial data that yields the exponential decay is not empty.

\section{Blow-up solution}

In this section, we deal with the blow-up solutions of problem (1.1). The basic idea comes from [30], however our argument contains nontrival modifications.

Lemma 4.1. Assume (G1), (1.2) and (1.3) hold. Let $u(x, t)$ be a local solution of problem (1.1) on $\left[0, T_{m}\right)$ with initial data $\left(u_{0}, u_{1}\right) \in H_{0}^{1}(\Omega) \times L^{2}(\Omega)$. If $T_{m}<\infty$, then

$$
\lim _{t \rightarrow T_{m}}\|u(t)\|_{q}=\infty \text { for all } q \geq 1 \text { such that } \frac{n(p-2)}{2}<q<p .
$$

Moreover, if $n \geq 3$ and $p=2 n /(n-2)=2^{*}(\omega>0)$, then (4.1) also holds for $q=p$.

Proof. From (3.7), we have

$$
\frac{1}{2}\left\|u_{t}(t)\right\|_{2}^{2}+\frac{l}{2}\|\nabla u(t)\|_{2}^{2} \leq \frac{1}{p}\|u(t)\|_{p}^{p}+E(0), \quad t \in\left[0, T_{m}\right),
$$

which, together with (3.2), implies

$$
\lim _{t \rightarrow T_{m}}\|u(t)\|_{p}=\infty
$$

This proves (4.1) at once for the case of $p=q=2 n /(n-2)$. For the remaining cases, notice (4.3) that implies 


$$
\lim _{t \rightarrow T_{m}}\|\nabla u(t)\|_{2}=\infty
$$

Moveover, by (4.2) we obtain

$$
l\|\nabla u(t)\|_{2}^{2} \leq \frac{2}{p}\|u(t)\|_{p}^{p}+2 E(0), \quad t \in\left[0, T_{m}\right) .
$$

From the Gagliardo-Nirenberg inequality we have

$$
\|u(t)\|_{p}^{p} \leq C\|u(t)\|_{q}^{p(1-\alpha)}\|\nabla u(t)\|_{2}^{p \alpha} \quad \text { for } \alpha=\frac{2 n(p-q)}{p(2 n+2 q-n q)}
$$

which yields

$$
\|\nabla u(t)\|_{2}^{2} \leq \frac{2}{l} E(0)+C_{1} C\|u(t)\|_{q}^{p(1-\alpha)}\|\nabla u(t)\|_{2}^{p \alpha} .
$$

Since $n(p-2) / 2<q<p$ implies $0<\alpha<1$ and $p \alpha<2$, the above inequality combined with (4.4) immediately yields (4.1).

Next we will prove the main blow-up result by the concavity method of Levine $[35,36]$ and the estimates similar as [30].

Theorem 4.2. Assume (G1), (G2), (1.2) and (1.3) hold. Let $u(x, t)$ be a local solution of problem (1.1) with initial data $\left(u_{0}, u_{1}\right) \in H_{0}^{1}(\Omega) \times L^{2}(\Omega)$. If $\omega>0$, then there is a real number $t_{0} \in\left[0, T_{m}\right)$ such that $u\left(t_{0}, \cdot\right) \in V_{k}$ and $E\left(t_{0}\right)<d_{k}$ if and only if $T_{m}<\infty$, where

$$
k=l-\frac{1}{p(p-2)} \int_{0}^{\infty} g(s) d s
$$

Proof. We first consider "if part", without loss of generality, we may assume that $t_{0}=$ 0 . Assume by contradiction that the solution $u$ is global. Then, for any $T>0$ we consider $H(t):[0, T] \rightarrow \mathbb{R}_{+}$defined by

$$
H(t)=\|u(t)\|_{2}^{2}+\int_{0}^{t}\|u(\tau)\|_{*}^{2} d \tau+(T-t)\left\|u_{0}\right\|_{*}^{2} .
$$

A direct computation yields

$$
H^{\prime}(t)=2 \int_{\Omega} u(t) u_{t}(t) d x+\|u(t)\|_{*}^{2}-\left\|u_{0}\right\|_{*}^{2}=2 \int_{\Omega} u(t) u_{t}(t) d x+2 \int_{0}^{t}\left(u(\tau), u_{t}(\tau)\right)_{*} d \tau
$$

and

$$
H^{\prime \prime}(t)=2\left\langle u_{t t}, u(t)\right\rangle+2\left\|u_{t}(t)\right\|_{2}^{2}+2\left(u(t), u_{t}(t)\right)_{* \prime} \quad \text { for a.e. } t \in[0, T] .
$$

By multiplying (1.1) by $u$ and integrating over $\Omega$, we have

$$
\left\langle u_{t t}, u(t)\right\rangle+\left(u(t), u_{t}(t)\right)_{*}=-\|\nabla u(t)\|_{2}^{2}-\int_{\Omega} \int_{0}^{t} g(t-\tau) \Delta u(\tau) d \tau u(t) d x+\|u(t)\|_{p}^{p},
$$


which implies

$$
H^{\prime \prime}(t)=2\left\|u_{t}(t)\right\|_{2}^{2}-2\|\nabla u(t)\|_{2}^{2}-2 \int_{\Omega} \int_{0}^{t} g(t-\tau) \Delta u(\tau) d \tau u(t) d x+2\|u(t)\|_{p}^{p} .
$$

Therefore, we have

$$
\begin{aligned}
& H(t) H^{\prime \prime}(t)-\frac{p+2}{4} H^{\prime}(t)^{2} \\
= & 2 H(t)\left(\left\|u_{t}(t)\right\|_{2}^{2}-\|\nabla u(t)\|_{2}^{2}-\int_{\Omega} \int_{0}^{t} g(t-\tau) \Delta u(\tau) d \tau u(t) d x+\|u(t)\|_{p}^{p}\right) \\
& -(p+2)\left(\int_{\Omega} u(t) u_{t}(t) d x+\int_{0}^{t}\left(u(\tau), u_{t}(\tau)\right)_{*} d \tau\right)^{2} \\
= & 2 H(t)\left(\left\|u_{t}(t)\right\|_{2}^{2}-\|\nabla u(t)\|_{2}^{2}-\int_{\Omega} \int_{0}^{t} g(t-\tau) \Delta u(\tau) d \tau u(t) d x+\|u(t)\|_{p}^{p}\right) \\
& +(p+2)\left(G(t)-\left(H(t)-(T-t)\left\|u_{0}\right\|_{*}^{2}\right)\left(\left\|u_{t}(t)\right\|_{2}^{2}+\int_{0}^{t}\left\|u_{t}(\tau)\right\|_{*}^{2} d \tau\right)\right),
\end{aligned}
$$

where $G(t):[0, T] \rightarrow \mathbb{R}_{+}$is the function defined by

$$
\begin{gathered}
G(t)=\left(\|u(t)\|_{2}^{2}+\int_{0}^{t}\|u(\tau)\|_{*}^{2} d \tau\right)\left(\left\|u_{t}(t)\right\|_{2}^{2}+\int_{0}^{t}\left\|u_{t}(\tau)\right\|_{*}^{2} d \tau\right) \\
-\left(\int_{\Omega} u(t) u_{t}(t) d x+\int_{0}^{t}\left(u(\tau), u_{t}(\tau)\right)_{*} d \tau\right)^{2} .
\end{gathered}
$$

Using the Schwarz inequality, we have

$$
\begin{gathered}
\left(\int_{\Omega} u(t) u_{t}(t) d x\right)^{2} \leq\|u(t)\|_{2}^{2}\left\|u_{t}(t)\right\|_{2^{\prime}}^{2} \\
\left(\int_{0}^{t}\left(u(\tau), u_{t}(\tau)\right)_{*} d \tau\right)^{2} \leq \int_{0}^{t}\|u(\tau)\|_{*}^{2} d \tau \int_{0}^{t}\left\|u_{t}(\tau)\right\|_{*}^{2} d \tau
\end{gathered}
$$

and

$$
\begin{aligned}
\int_{\Omega} u(t) u_{t}(t) d x \int_{0}^{t}\left(u(\tau), u_{t}(\tau)\right)_{*} d \tau & \leq\|u(t)\|_{2}\left(\int_{0}^{t}\left\|u_{t}(\tau)\right\|_{*}^{2} d \tau\right)^{\frac{1}{2}}\left\|u_{t}(t)\right\|_{2}\left(\int_{0}^{t}\|u(\tau)\|_{*}^{2} d \tau\right)^{\frac{1}{2}} \\
& \leq \frac{1}{2}\|u(t)\|_{2}^{2} \int_{0}^{t}\left\|u_{t}(\tau)\right\|_{*}^{2} d \tau+\frac{1}{2}\left\|u_{t}(t)\right\|_{2}^{2} \int_{0}^{t}\|u(\tau)\|_{*}^{2} d \tau .
\end{aligned}
$$


These three inequalities entail $G(t) \geq 0$ for every $[0, T]$. Using (4.6), we get

$$
H(t) H^{\prime \prime}(t)-\frac{p+2}{4} H^{\prime}(t)^{2} \geq H(t) L(t) \quad \text { for a.e. } t \in[0, T]
$$

where

$$
\begin{aligned}
L(t)= & -p\left\|u_{t}(t)\right\|_{2}^{2}-2\|\nabla u(t)\|_{2}^{2}+2\|u(t)\|_{p}^{p}-(p+2) \int_{0}^{t}\left\|u_{t}(t)\right\|_{*}^{2} d \tau \\
& -2 \int_{\Omega} \int_{0}^{t} g(t-\tau) \Delta u(\tau) d \tau u(t) d x .
\end{aligned}
$$

For the last term on the left of (4.8), we have

$$
\begin{aligned}
& -\int_{\Omega} \int_{0}^{t} g(t-\tau) \Delta u(\tau) d \tau u(t) d x=\int_{0}^{t} g(t-\tau) \int_{\Omega} \nabla u(\tau) \nabla u(\tau) d x d \tau \\
= & \int_{0}^{t} g(t-\tau) \int_{\Omega} \nabla u(t) \nabla(u(\tau)-u(\tau)) d x d \tau+\int_{0}^{t} g(t-\tau)\|\nabla u(t)\|_{2}^{2} d \tau \\
= & \int_{0}^{t} g(t-\tau) \int_{\Omega} \nabla u(t) \nabla(u(\tau)-u(\tau)) d x d \tau+\int_{0}^{t} g(\tau)\|\nabla u(t)\|_{2}^{2} d \tau .
\end{aligned}
$$

Combining (4.8) with (4.9), we get

$$
\begin{aligned}
L(t)= & -p\left\|u_{t}(t)\right\|_{2}^{2}-2\left(1-\int_{0}^{t} g(\tau) d \tau\right)\|\nabla u(t)\|_{2}^{2}+2\|u(t)\|_{p}^{p} \\
& +2 \int_{0}^{t} g(t-\tau) \int_{\Omega} \nabla u(t) \nabla(u(\tau)-u(\tau)) d x d \tau-(p+2) \int_{0}^{t}\left\|u_{t}(\tau)\right\|_{*}^{2} d \tau \\
\geq & -p\left\|u_{t}(t)\right\|_{2}^{2}-2\left(1-\int_{0}^{t} g(\tau) d \tau\right)\|\nabla u(t)\|_{2}^{2}+2\|u(t)\|_{p}^{p}-2\left(\frac{p}{2} \int_{0}^{t} g(t-\tau) \mid \nabla u(\tau)\right. \\
& \left.-\left.\nabla u(t)\right|^{2} d \tau+\frac{1}{2 p} \int_{0}^{t} g(\tau)\|\nabla u(t)\|_{2}^{2} d \tau\right)-(p+2) \int_{0}^{t}\left\|u_{t}(\tau)\right\|_{*}^{2} d \tau \\
\geq & -2 p E(t)+(p-2)\left(1-\int_{0}^{t} g(\tau) d \tau\right)\|\nabla u(t)\|_{2}^{2}-\frac{1}{p} \int_{0}^{t} g(\tau)\|\nabla u(t)\|_{2}^{2} d \tau \\
& -(p+2) \int_{0}^{t}\left\|u_{t}(\tau)\right\|_{*}^{2} d \tau .
\end{aligned}
$$

Using (3.7), we have

$$
E(t)+\int_{0}^{t}\left\|u_{t}(\tau)\right\|_{*}^{2} d \tau \leq E(0)
$$


and then

$$
\begin{aligned}
L(t) \geq & -2 p E(0)+(p-2)\left(1-\int_{0}^{t} g(\tau) d \tau\right)\|\nabla u(t)\|_{2}^{2}-\frac{1}{p} \int_{0}^{t} g(\tau)\|\nabla u(t)\|_{2}^{2} d \tau \\
& +(p-2) \int_{0}^{t}\left\|u_{t}(\tau)\right\|_{*}^{2} d \tau \\
\geq & -2 p E(0)+(p-2) \int_{0}^{t}\left\|u_{t}(\tau)\right\|_{*}^{2} d \tau+\left((p-2)\left(1-\int_{0}^{t} g(\tau) d \tau\right)-\frac{1}{p} \int_{0}^{t} g(\tau) d \tau\right)\|\nabla u(t)\|_{2}^{2} \\
\geq & 2 p\left(\frac{p-2}{2 p}\left(\left(1-\int_{0}^{t} g(\tau) d \tau\right)-\frac{1}{p(p-2)} \int_{0}^{t} g(\tau) d \tau\right)\|\nabla u(t)\|_{2}^{2}-E(0)\right) \\
& +(p-2) \int_{0}^{t}\left\|u_{t}(\tau)\right\|_{*}^{2} d \tau \\
\geq & 2 p\left(\frac{p-2}{2 p}\left(l-\frac{1}{p(p-2)} \int_{0}^{t} g(\tau) d \tau\right)\|\nabla u(t)\|_{2}^{2}-E(0)\right)+(p-2) \int_{0}^{t}\left\|u_{t}(\tau)\right\|_{*}^{2} d \tau \\
\geq & 2 p\left(\frac{p-2}{2 p} k\|\nabla u(t)\|_{2}^{2}-E(0)\right)+(p-2) \int_{0}^{t}\left\|u_{t}(\tau)\right\|_{*}^{2} d \tau .
\end{aligned}
$$

Since

$$
\int_{0}^{\infty} g(s) d s<\frac{p / 2-1}{p / 2-1+1 /(2 p)}
$$

we have

$$
0<k=l-\frac{1}{p(p-2)} \int_{0}^{\infty} g(\tau) d \tau \leq l
$$

By Lemma 3.4, we have

$$
u(t, \cdot) \in V_{k} \text { and } E(t)<d_{k} \quad \text { for } t \in\left[0, T_{m}\right) .
$$

Then, we have

$$
d_{k} \leq \frac{p-2}{2 p} k^{\frac{p}{p-2}} \frac{\|\nabla u(t)\|_{2}^{\frac{2 p}{p-2}}}{\|u(t)\|_{p}^{\frac{2 p}{p-2}}} \leq \frac{p-2}{2 p} k\|\nabla u(t)\|_{2}^{2}, \quad \text { for } t \in\left[0, T_{m}\right) .
$$

The above inequality comes from [29]; see [28,29] for further details. Since $E(0)<d_{k}$, there exists $\delta>0$ (independent of $T$ ) such that

$$
L(t) \geq \delta, \quad \text { for } t \in[0, T] .
$$

From (4.10) and the definition of $H(t)$, there also exists $\rho>0$ (independent of $T$ ) such that

$$
H(t) \geq \rho, \quad \text { for } t \in[0, T]
$$


By (4.7), (4.11), and (4.12) it follows that

$$
H(t) H^{\prime \prime}(t)-\frac{p+2}{4} H^{\prime}(t)^{2} \geq \delta \rho, \quad \text { for a.e. } t \in[0, T] .
$$

Setting $y(t)=H(t)^{-(p-2) / 4}$, then we have

$$
\gamma^{\prime}(t) \leq \frac{p-2}{4} \delta \rho y(t)^{\frac{p+6}{p-2}}, \quad \text { for a.e. } t \in[0, T]
$$

which implies that $y(t)$ reaches 0 in finite time, say as $t \rightarrow T^{*}$. Since $T^{*}$ is independent of the initial choice of $T$, we may assume that $T^{*}<T$. This tells us that

$$
\lim _{t \rightarrow T *} H(t)=\infty
$$

In turn, this implies that

$$
\lim _{t \rightarrow T *}\|\nabla u(t)\|_{2}^{2}=\infty
$$

Indeed, if $\|u(t)\|_{2}^{2} \rightarrow \infty$ as $t \rightarrow T^{*}$, then (4.13) immediately follows. On the contrary, if $\|u(t)\|_{2}^{2}$ remains bounded on $\left[0, T^{*}\right)$, then

$$
\lim _{t \rightarrow T *} \int_{0}^{t}\|u(\tau)\|_{*}^{2} d \tau=\infty
$$

so that again (4.13) is satisfied. This implies a contradiction, i,e., $T_{m}<\infty$.

Conversely, for "only if part" we assume now that $T_{m}<\infty$. Notice first that, for every $t>0$, there holds

$$
\int_{0}^{t}\left\|u_{t}(\tau)\right\|_{*}^{2} d \tau \geq \frac{1}{t}\left(\int_{0}^{t}\left\|u_{t}(\tau)\right\|_{*} d \tau\right)^{2} \geq \frac{1}{t}\left(\|u(t)\|_{*}-\|u\|_{*}\right)^{2} .
$$

Hence, by (3.7) and $0<k \leq l$, we have

$$
\frac{1}{2} I_{k}(u(t)) \leq E(t) \leq E(0)-\frac{1}{t}\left(\|u(t)\|_{*}-\|u\|_{*}\right)^{2} .
$$

By Lemma 4.1 we have $\|\nabla u(t)\|_{2} \rightarrow \infty$ as $t \rightarrow T_{m}$, i.e., $\|u(t)\|_{*} \rightarrow \infty$ as $t \rightarrow T_{m}$, together with (4.14) which implies

$$
\lim _{t \rightarrow T_{m}} I_{k}(u(t))=\lim _{t \rightarrow T_{m}} E(t)=-\infty .
$$

Since $J_{k}(u(t)) \leq E(t)$, by (4.15) we obtain that

$$
J_{k}\left(u\left(t_{0}\right)\right) \leq E\left(t_{0}\right)<d_{k} \quad I_{k}\left(u\left(t_{0}\right)\right)<0
$$

for some $t_{0} \in\left[0, T_{m}\right)$. These imply $u\left(t_{0}\right) \in V_{k}, E\left(t_{0}\right)<d_{k}$.

Remark 4.1. The "if part" of Theorem 4.2 means that the solution to (1.1) blows up in a finite time for suitable "large" initial data $u_{0}$ and $u_{1}$ in the sense of $u_{0} \in V_{k}$ and $E$ $(0)<d_{k}$. Also, (4.15) is an essential behavior for which the solution of (1.1) blows up in a finite time. 
Remark 4.2. In Theorem 4.2, we restrict $\omega>0$ in order to prove the "only if part". In fact, if $\omega>0$, it is easy to obtain $\|u(t)\|_{*} \rightarrow \infty$ as $t \rightarrow T_{m}$ from $\|\nabla u(t)\|_{2} \rightarrow \infty$ as $t \rightarrow$ $T_{m}$, which implies $E(t) \rightarrow-\infty$ as $t \rightarrow T_{m}$. If $\omega=0$ (only with weak damping), assuming $2<p \leq 2+2 / n$, then we can obtain $\|u(t)\|_{2} \rightarrow \infty$ as $t \rightarrow T_{m}$ (see [37] for details) which yields that Theorem 4.2 also holds for the case of $\omega=0$ with $2<p \leq 2+2 / n$.

Next, we consider the blow-up solution of problem (1.1) for the case of weak damping $(\omega=0)$ with arbitrary positive initial energy. We need an addition assumption on the relaxation function $g$ :

(G3) The function of $e^{\frac{t}{2}} g(t)$ is of positive in the following sense:

$$
\int_{0}^{t} v(s) \int_{0}^{s} e^{\frac{s-r}{2}} g(s-\tau) v(\tau) d \tau d s \geq 0,
$$

$\forall v \in C^{1}([0, \infty))$ and $\forall t>0$.

Obviously, $g(t)=\varepsilon e^{-t}$ with $0<\varepsilon<1$ satisfies assumptions (G1)-(G3). Let

$$
I(u)=I_{1}(u)=\|\nabla u\|_{2}^{2}-\|u\|_{p}^{p} .
$$

Lemma 4.3. [27, Lemma 2.1]) Assume that $g(t)$ satisfies (G1), (G3) and $\Lambda(t)$ is a function that is twice continuously differentiable, satisfying

$$
\left\{\begin{array}{l}
\Lambda^{\prime \prime}(t)+\mu \Lambda^{\prime}(t)>\int_{0}^{t} g(t-\tau) \int_{\Omega} \nabla u(\tau) \nabla u(t) d x d \tau, \\
\Lambda(0)>, \quad \Lambda(0)>0,
\end{array}\right.
$$

for every $t \mathrm{~L}\left[0, T_{m}\right)$, where $u(t)$ is the corresponding solution of problem (1.1) with weak damping. Then the function $\Lambda(t)$ is strictly increasing on $\left[0, T_{m}\right)$.

Lemma 4.4. Suppose that $u_{0} \in H_{0}^{1}(\Omega), u_{1} \in L^{2}(\Omega)$ satisfy

$$
\int_{\Omega} u_{0}(x) u_{1}(x) d x>0 .
$$

If the local solution $u(t)$ of problem (1.1) with weak damping exists on $\left[0, T_{m}\right)$ and satisfies $I(u(t))<0$, then $\|u(t)\|_{2}^{2}$ is strictly increasing on $\left[0, T_{m}\right)$.

Proof. Since $u(t)$ is the local solution of problem (1.1) with weak damping, by a simple computation we have

$$
\begin{aligned}
\frac{1}{2} \frac{d^{2}}{d t^{2}}\|u(t)\|_{2}^{2} & =\int_{\Omega}\left(\left|u_{t}(t)\right|^{2}+u u_{t t}\right) d x \\
& =\left\|u_{t}(t)\right\|_{2}^{2}-\mu \int_{\Omega} u u_{t} d x-I(u(t))+\int_{0}^{t} g(t-\tau) \int_{\Omega} \nabla u(\tau) \nabla u(t) d x d \tau \\
& \geq-\mu \int_{\Omega} u u_{t} d x+\int_{0}^{t} g(t-\tau) \int_{\Omega} \nabla u(\tau) \nabla u(t) d x d \tau
\end{aligned}
$$

where the last inequality uses $I(u(t))<0$. Then we get

$$
\frac{d^{2}}{d t^{2}}\|u(t)\|_{2}^{2}+\mu \frac{d}{d t}\|u(t)\|_{2}^{2}>\int_{0}^{t} g(t-\tau) \int_{\Omega} \nabla u(\tau) \nabla u(t) d x d \tau .
$$


Therefore, this lemma comes from Lemma 4.3.

Theorem 4.5. Assume (G1), (G3), (1.2) and (1.3) hold. Let $u(x, t)$ be a local solution of problem (1.1) with initial data $\left(u_{0}, u_{1}\right) \in H_{0}^{1}(\Omega) \times L^{2}(\Omega)$. If $\omega=0, g(s)$ also satisfies

$$
\int_{0}^{\infty} g(s) d s<\frac{(p-2)^{2}}{(p-1)^{2}}
$$

and $\left(u_{0}, u_{1}\right)$ satisfies the following conditions

$$
E(0)>0, \quad \int_{\Omega} u_{0}(x) u_{1}(x) d x>0, \quad I\left(u_{0}\right)<0, \quad\left\|u_{0}\right\|_{2}^{2}>\frac{2 p E(0)}{(k p-2) \lambda} .
$$

where

$$
k=l-\frac{1}{p(p-2)} \int_{0}^{\infty} g(s) d s>0
$$

and $\lambda$ is the first eigenvalue of the operator $-\Delta$ under homogeneous Dirichlet boundary conditions, then the corresponding solution $u(t)$ of problem (1.1) blows up in a finite time, i.e., $T_{m}<\infty$.

Proof. Without loss of generality, we may assume $\mu=1$. First, by a contradiction argument we claim that

$$
I(u(t))<0,
$$

and

$$
\|u(t)\|_{2}^{2}>\frac{2 p E(0)}{(k p-2) \lambda}
$$

for every $t \in\left[0, T_{m}\right)$. If this was not the case, then there would exist a time $t_{1}$ such that

$$
t_{1}=\min \left\{t \in\left(0, T_{m}\right): I(u(t))=0\right\}>0 .
$$

By the continuity of the solution $u(t)$ as a function of $t$, we see that $I(u(t))<0$ when $t$ $\in\left(0, t_{1}\right)$ and $I(u(t))=0$. Thus by Lemma 4.4 we have

$$
\|u(t)\|_{2}^{2}>\left\|u_{0}\right\|_{2}^{2}>\frac{2 p E(0)}{(k p-2) \lambda}
$$

for every $t \in\left[0, t_{1}\right)$. In addition, it is obvious that $\|u(t)\|_{2}^{2}$ is continuous on $\left[0, t_{1}\right]$. Thus the following inequality is obtained:

$$
\left\|u\left(t_{1}\right)\right\|_{2}^{2}>\frac{2 p E(0)}{(k p-2) \lambda} .
$$

On the other hand, it follows from the definition of $E(t)$ and (3.7) that

$$
\frac{1}{2}\left(1-\int_{0}^{t_{1}} g(s) d s\right)\left\|\nabla u\left(t_{1}\right)\right\|_{2}^{2}-\frac{1}{p}\|u(t)\|_{p}^{p} \leq E(0) .
$$


Since $0<k \leq l \leq 1-\int_{0}^{t_{1}} g(s) d s$, from (4.22), we have

$$
\frac{k}{2}\left\|\nabla u\left(t_{1}\right)\right\|_{2}^{2}-\frac{1}{p}\|u(t)\|_{p}^{p} \leq E(0)
$$

Noting the fact that $I\left(u\left(t_{1}\right)\right)=0$, we then have

$$
\frac{k p-2}{2 p}\left\|\nabla u\left(t_{1}\right)\right\|_{2}^{2} \leq E(0)
$$

Thus, by the Poincaré inequality and (4.16) we have

$$
\left\|u\left(t_{1}\right)\right\|_{2}^{2} \leq \frac{2 p E(0)}{(k p-2) \lambda} .
$$

Obviously, there is a contradiction between (4.21) and (4.23). Thus, we have proved that (4.18) is true for every for every $t \in\left[0, T_{m}\right)$. Furthermore, by Lemma 4.4 we see that (4.19) is also valid on $t \in\left[0, T_{m}\right)$.

Secondly, we prove that the solution of problem (1.1) blows up in a finite time. The proof is similar "if part" in the Theorem 4.2, for the convenience of the readers, we give the sketch of the proof here. Assume by contradiction that the solution $u$ is global. Then, for sufficiently large $T>0$ we consider $\Phi(t):[0, T] \rightarrow \mathbb{R}_{+}$defined by

$$
\Phi(t)=\|u(t)\|_{2}^{2}+\int_{0}^{t}\|u(\tau)\|_{2}^{2} d \tau+(T-t)\left\|u_{0}\right\|_{2}^{2}
$$

A direct computation yields

$$
\Phi^{\prime}(t)=2 \int_{\Omega} u(t) u_{t}(t) d x+\|u(t)\|_{2}^{2}-\left\|u_{0}\right\|_{2}^{2}=2 \int_{\Omega} u(t) u_{t}(t) d x+2 \int_{0}^{t}\left(u(\tau), u_{t}(\tau)\right) d \tau
$$

and

$$
\Phi^{\prime \prime}(t)=2\left\langle u_{t t}, u(t)\right\rangle+2\left\|u_{t}(t)\right\|_{2}^{2}+2\left(u(t), u_{t}(t)\right), \quad \text { for a.e. } t \in[0, T]
$$

where $\left(u(t), u_{t}(t)\right)=\int_{\Omega} u(t) u_{t}(t) d x$. By multiplying (1.1) by $u$ and integrating over $\Omega$, we have

$$
\left\langle u_{t t}, u(t)\right\rangle+\left(u(t), u_{t}(t)\right)=-\|\nabla u(t)\|_{2}^{2}-\int_{\Omega} \int_{0}^{t} g(t-\tau) \Delta u(\tau) d \tau u(t) d x+\|u(t)\|_{p^{\prime}}^{p}
$$

which implies

$$
\Phi^{\prime \prime}(t)=2\left\|u_{t}(t)\right\|_{2}^{2}-2\|\nabla u(t)\|_{2}^{2}-2 \int_{\Omega} \int_{0}^{t} g(t-\tau) \Delta u(\tau) d \tau u(t) d x+2\|u(t)\|_{p}^{p} .
$$


Therefore, we have

$$
\begin{aligned}
& \Phi(t) \Phi^{\prime \prime}(t)-\frac{p+2}{4} \Phi^{\prime}(t)^{2} \\
= & 2 \Phi(t)\left(\left\|u_{t}(t)\right\|_{2}^{2}-\|\nabla u(t)\|_{2}^{2}-\int_{\Omega} \int_{0}^{t} g(t-\tau) \Delta u(\tau) d \tau u(t) d x+\|u(t)\|_{p}^{p}\right) \\
& -(p+2)\left(\int_{\Omega} u(t) u_{t}(t) d x+\int_{0}^{t}\left(u(\tau), u_{t}(\tau)\right) d \tau\right)^{2} \\
= & 2 \Phi(t)\left(\left\|u_{t}(t)\right\|_{2}^{2}-\|\nabla u(t)\|_{2}^{2}-\int_{\Omega} \int_{0}^{t} g(t-\tau) \Delta u(\tau) d \tau u(t) d x+\|u(t)\|_{p}^{p}\right) \\
& +(p+2)\left(\Psi(t)-\left(\Phi(t)-(T-t)\left\|u_{0}\right\|_{2}^{2}\right)\left(\left\|u_{t}(t)\right\|_{2}^{2}+\int_{0}^{t}\left\|u_{t}(\tau)\right\|_{2}^{2} d \tau\right)\right),
\end{aligned}
$$

where $\Psi(t):[0, T] \rightarrow \mathbb{R}_{+}$is the function defined by

$$
\begin{aligned}
\Psi(t)= & \left(\|u(t)\|_{2}^{2}+\int_{0}^{t}\|u(\tau)\|_{2}^{2} d \tau\right)\left(\left\|u_{t}(t)\right\|_{2}^{2}+\int_{0}^{t}\left\|u_{t}(\tau)\right\|_{2}^{2} d \tau\right) \\
& -\left(\int_{\Omega} u(t) u_{t}(t) d x+\int_{0}^{t}\left(u(\tau), u_{t}(\tau)\right) d \tau\right)^{2} .
\end{aligned}
$$

Using the Schwarz inequality, we have

$$
\begin{gathered}
\left(\int_{\Omega} u(t) u_{t}(t) d x\right)^{2} \leq\|u(t)\|_{2}^{2}\left\|u_{t}(t)\right\|_{2}^{2}, \\
\left(\int_{0}^{t}\left(u(\tau), u_{t}(\tau)\right) d \tau\right)^{2} \leq \int_{0}^{t}\|u(\tau)\|_{2}^{2} d \tau \int_{0}^{t}\left\|u_{t}(\tau)\right\|_{2}^{2} d \tau,
\end{gathered}
$$

and

$$
\begin{aligned}
\int_{\Omega} u(t) u_{t}(t) d x+\int_{0}^{t}\left(u(\tau), u_{t}(\tau)\right) d \tau & \leq\|u(t)\|_{2}\left(\int_{0}^{t}\left\|u_{t}(\tau)\right\|_{2}^{2} d \tau\right)^{\frac{1}{2}}\left\|u_{t}(t)\right\|_{2}\left(\int_{0}^{t}\|u(\tau)\|_{2}^{2} d \tau\right)^{\frac{1}{2}} \\
& \leq \frac{1}{2}\|u(t)\|_{2}^{2} \int_{0}^{t}\left\|u_{t}(\tau)\right\|_{2}^{2} d \tau+\frac{1}{2}\left\|u_{t}(t)\right\|_{2}^{2} \int_{0}^{t}\|u(\tau)\|_{2}^{2} d \tau .
\end{aligned}
$$

These three inequalities entail $\Psi(t) \geq 0$ for every $[0, T]$. Using (4.24), we get

$$
\Phi(t) \Phi^{\prime \prime}(t)-\frac{p+2}{4} \Phi^{\prime}(t)^{2} \geq \Phi(t) \Upsilon(t) \quad \text { for a.e. } t \in[0, T],
$$


where

$$
\begin{aligned}
\Upsilon(t)=-p\left\|u_{t}(t)\right\|_{2}^{2}-2\|\nabla u(t)\|_{2}^{2}+2\|u(t)\|_{p}^{p}-(p+2) \int_{0}^{t}\left\|u_{t}(\tau)\right\|_{2}^{2} d \tau \\
-2 \int_{\Omega} \int_{0}^{t} g(t-\tau) \Delta u(\tau) d \tau u(t) d x .
\end{aligned}
$$

Combining (4.9) with (4.26), we get

$$
\begin{aligned}
\Upsilon(t)= & -p\left\|u_{t}(t)\right\|_{2}^{2}-2\left(1-\int_{0}^{t} g(\tau) d \tau\right)\|\nabla u(t)\|_{2}^{2}+2\|u(t)\|_{p}^{p} \\
& +2 \int_{0}^{t} g(t-\tau) \int_{\Omega} \nabla u(t) \nabla(u(\tau)-u(t)) d x d \tau-(p+2) \int_{0}^{t}\left\|u_{t}(\tau)\right\|_{2}^{2} d \tau \\
\geq & -p\left\|u_{t}(t)\right\|_{2}^{2}-2\left(1-\int_{0}^{t} g(\tau) d \tau\right)\|\nabla u(t)\|_{2}^{2}+2\|u(t)\|_{p}^{p}-2\left(\frac{p}{2} \int_{0}^{t} g(t-\tau) \mid \nabla u(\tau)\right. \\
& \left.-\left.\nabla u(t)\right|^{2} d \tau+\frac{1}{2 p} \int_{0}^{t} g(\tau)\|\nabla u(t)\|_{2}^{2} d \tau\right)-(p+2) \int_{0}^{t}\left\|u_{t}(\tau)\right\|_{2}^{2} d \tau \\
\geq & -2 p E(t)+(p-2)\left(1-\int_{0}^{t} g(\tau) d \tau\right)\|\nabla u(t)\|_{2}^{2}-\frac{1}{p} \int_{0}^{t} g(\tau)\|\nabla u(t)\|_{2}^{2} d \tau \\
& -(p+2) \int_{0}^{t}\left\|u_{t}(\tau)\right\|_{2}^{2} d \tau .
\end{aligned}
$$

Using (3.7) for $\omega=0$, we have

$$
E(t)+\int_{0}^{t}\left\|u_{t}(\tau)\right\|_{2}^{2} d \tau \leq E(0),
$$

and then

$$
\begin{aligned}
\Upsilon(t) \geq & -2 p E(0)+(p-2)\left(1-\int_{0}^{t} g(\tau) d \tau\right)\|\nabla u(t)\|_{2}^{2}-\frac{1}{p} \int_{0}^{t} g(\tau)\|\nabla u(t)\|_{2}^{2} d \tau \\
& -(p+2) \int_{0}^{t}\left\|u_{t}(\tau)\right\|_{2}^{2} d \tau \\
\geq & -2 p E(0)+(p-2) \int_{0}^{t}\left\|u_{t}(\tau)\right\|_{2}^{2} d \tau+\left((p-2)\left(1-\int_{0}^{t} g(\tau) d \tau\right)-\frac{1}{p} \int_{0}^{t} g(\tau) d \tau\right)\|\nabla u(t)\|_{2}^{2} \\
\geq & 2 p\left(\frac{p-2}{2 p}\left(\left(1-\int_{0}^{t} g(\tau) d \tau\right)-\frac{1}{p(p-2)} \int_{0}^{t} g(\tau) d \tau\right)\|\nabla u(t)\|_{2}^{2}-E(0)\right) \\
& +(p-2) \int_{0}^{t}\left\|u_{t}(\tau)\right\|_{2}^{2} d \tau \\
\geq & 2 p\left(\frac{p-2}{2 p}\left(l-\frac{1}{p(p-2)} \int_{0}^{t} g(\tau) d \tau\right)\|\nabla u(t)\|_{2}^{2}-E(0)\right)+(p-2) \int_{0}^{t}\left\|u_{t}(\tau)\right\|_{2}^{2} d \tau \\
\geq & 2 p\left(\frac{p-2}{2 p} k\|\nabla u(t)\|_{2}^{2}-E(0)\right)+(p-2) \int_{0}^{t}\left\|u_{t}(\tau)\right\|_{2}^{2} d \tau \\
\geq & 2 p\left(\frac{p-2}{2 p} \lambda k\left\|u_{0}\right\|_{2}^{2}-E(0)\right)+(p-2) \int_{0}^{t}\left\|u_{t}(\tau)\right\|_{2}^{2} d \tau
\end{aligned}
$$


where the last inequality follows from Lemma 4.4 and the Poincaré inequality. Since $0<k<1$, we have $p k-2<(p-2) k$. From (4.17), we get

$$
\frac{(p-2) k}{2 p} \lambda\left\|u_{0}\right\|_{2}^{2}-E(0)>\frac{p k-2}{2 p} \lambda\left\|u_{0}\right\|_{2}^{2}-E(0)>0 .
$$

Therefore, there exists $\delta_{1}>0$ (independent of $T$ ) such that

$$
\Upsilon(t) \geq \delta_{1} \quad \text { for } t \in[0, T]
$$

From Lemma 4.4, (4.17) and the definition of $\Phi(t)$, there also exists $\rho_{1}>0$ (independent of $T$ ) such that

$$
\Phi(t) \geq \rho_{1} \quad \text { for } t \in[0, T] .
$$

By (4.24), (4.27), and (4.28) it follows that

$$
\Phi(t) \Phi^{\prime \prime}(t)-\frac{p+2}{4} \Phi^{\prime}(t)^{2} \geq \delta_{1} \rho_{1} \quad \text { for a.e. } t \in[0, T] .
$$

The rest of the proof is the same as "if part" in the Theorem 4.2, so we omit it here.

\section{The boundedness of global solution}

In this section, we will prove the boundedness of global solutions $u(t)$ to problem (1.1) for strong $(\omega>0)$ damping, namely,

$$
u \in L^{\infty}\left(\mathbb{R}^{+} ; H_{0}^{1}(\Omega)\right) \cap W^{1, \infty}\left(\mathbb{R}^{+} ; L^{2}(\Omega)\right) .
$$

Throughout this section, we assume that

$$
E\left(u(t), u_{t}(t)\right)=E(t) \geq d_{k} \quad \text { for all } t \geq 0 .
$$

If (5.2) holds, then the solution to problem (1.1) for strong $(\omega>0)$ damping is global. Indeed, if $u(t)$ blows up in finite time, by Theorem 4.2, $E\left(t_{0}\right)<d_{k}$ for some $t_{0}>0$. Hence, $E\left(u(t), u_{t}(t)\right)=E(t)<d_{k}$ for all $t \geq t_{0}$. This is a contradiction.

Since $u_{t}(t) \in H_{0}^{1}(\Omega)$ for a.e. $t \geq 0$, we combine Poincare inequality with (3.7) and (5.2) to show that, for every $t>0$ we have

$$
\int_{0}^{t}\left\|u_{t}(\tau)\right\|_{2}^{2} d \tau \leq C \int_{0}^{t}\left\|u_{t}(\tau)\right\|_{*}^{2} d \tau \leq C\left(E(0)-d_{k}\right) .
$$

Letting $t \rightarrow \infty$, we conclude that

$$
\int_{0}^{\infty}\left\|u_{t}(\tau)\right\|_{2}^{2} d \tau<\infty, \quad \int_{0}^{\infty}\left\|\nabla u_{t}(\tau)\right\|_{2}^{2} d \tau<\infty .
$$

Furthermore, observe that by the definition of $E(t)$, we have

$$
\|u(t)\|_{p}^{p} \geq \frac{p l}{2}\|\nabla u(t)\|_{2}^{2}-p E(0)
$$


Since

$$
\left\langle u_{t t}, u(t)\right\rangle=\frac{d}{d t} \int_{\Omega} u_{t}(t) u(t) d x-\left\|u_{t}(t)\right\|_{2}^{2} \text { for a.e. } t \in[0, \infty]
$$

from (4.5), we have

$$
\frac{d}{d t}\left(\int_{\Omega} u_{t}(t) u(t) d x+\frac{1}{2}\|u(t)\|_{*}^{2}\right)=\left\|u_{t}\right\|_{2}^{2}-\|\nabla u\|_{2}^{2}+\|u\|_{p}^{p}-\int_{\Omega} \int_{0}^{t} g(t-\tau) \Delta u(\tau) d \tau u(t) d x
$$

Combining (4.9) with (5.5), we get

$$
\begin{aligned}
& \frac{d}{d t}\left(\int_{\Omega} u_{t}(t) u(t) d x+\frac{1}{2}\|u(t)\|_{*}^{2}\right) \\
= & \left\|u_{t}\right\|_{2}^{2}-\|\nabla u\|_{2}^{2}+\|u\|_{p}^{p}+\int_{0}^{t} g(\tau)\|\nabla u(t)\|_{2}^{2} d \tau \\
& +\int_{0}^{t} g(t-\tau) \int_{\Omega} \nabla u(t) \nabla(u(\tau)-u(t)) d x d \tau \\
\geq & \left\|u_{t}\right\|_{2}^{2}-\|\nabla u\|_{2}^{2}+\|u\|_{p}^{p}+\frac{1}{2} \int_{0}^{t} g(\tau)\|\nabla u(t)\|_{2}^{2} d \tau-\frac{1}{2}(g \circ \nabla u)(\tau) \\
\geq & \left(1-\frac{1}{p}\right)\|u\|_{p}^{p}-\frac{1}{2}\|\nabla u\|_{2}^{2}-E(t) \geq \frac{(p-1) l-1}{2}\|\nabla u\|_{2}^{2}-p E(0),
\end{aligned}
$$

where the last inequality follows from (5.4).

Inspired by Gazzola and Weth [38] we now prove a crucial stability result.

Lemma 5.1. Assume (G1), (G2), (1.2) and (1.3) hold. If $u(t)$ is a solution to problem (1.1) for strong $(\omega>0)$ damping satisfying $E\left(u(t), u_{t}(t)\right)=E(t) \geq d_{k}$ for all $t \geq 0$, then we have

$$
\lim _{t \rightarrow \infty}\|\nabla u(t)-\nabla u(t+\eta)\|_{2}=0, \quad \forall \eta>0 .
$$

Proof. Fixed $\eta>0$, by (3.7), for every $t>0$ we have

$$
\begin{aligned}
\int_{\Omega} \nabla u(t)-\left.\nabla u(t+\eta)\right|^{2} d x & =\int_{\Omega}\left|\int_{t}^{t+\eta} \nabla u_{t}(\tau) d \tau\right|^{2} \leq \eta \int_{\Omega} \int_{t}^{t+\eta}\left|\nabla u_{t}(\tau)\right|^{2} d \tau \\
& \leq C \eta \int_{0}^{t+\eta}\left\|u_{t}(\tau)\right\|_{*}^{2} d \tau \leq C \eta(E(t)-E(t+\eta)) .
\end{aligned}
$$

Since $E(t)$ is nonincreasing and lower bounded by $d_{k}, E(t)$ admits finite limit as $t \rightarrow \infty$. This immediately yields the assertion by letting $t \rightarrow \infty$ in the previous inequality.

Theorem 5.2. Assume (G1), (1.2) and (1.3) hold. In addition, g(s) also satisfies

$$
\int_{0}^{\infty} g(s) d s<\frac{p-2}{p-1}
$$


If $u(t)$ is a solution to problem (1.1) for strong $(\omega>0)$ damping satisfying $E\left(u(t), u_{t}(t)\right)$ $=E(t) \geq d_{k}$ for all $t \geq 0$, then the solution $u(t)$ satisfies (5.1).

Proof. Assuming by contradiction that (5.1) fails, namely that there exists a diverging sequence $t_{j} \subset \mathbb{R}_{+}$such that

$$
\left\|u_{t}\left(t_{j}\right)\right\|_{2}^{2}+\left\|\nabla u\left(t_{j}\right)\right\|_{2}^{2} \rightarrow \infty \quad \text { as } j \rightarrow \infty .
$$

Then, by the definition of $E(t)$ and (5.2), we have $\left\|u\left(t_{j}\right)\right\|_{p} \rightarrow \infty$ as $j \rightarrow \infty$. By Sobolev inequality we get

$$
\left\|\nabla u\left(t_{j}\right)\right\|_{2} \rightarrow \infty \quad \text { as } j \rightarrow \infty .
$$

By (5.9) and continuity, we can select a diverging sequence $\bar{t}_{m} \subset \mathbb{R}_{+}$such that $\left\|u\left(\bar{t}_{m}\right)\right\|_{*}^{2}=m^{2}+1$. Moreover, by Lemma 5.1, we have

$$
\lim _{\tau \rightarrow \infty} \sup \left\{\eta>0:\|u(t)-u(t+\eta)\|_{*}<1, \quad \forall t \geq \tau\right\}=\infty .
$$

Then, we find a second diverging sequence $\tau_{m} \subset \mathbb{R}_{+}$such that

$$
m^{2} \leq\|u(t)\|_{*}^{2} \leq m^{2}+2 \quad \text { for every } t \in\left[\bar{t}_{m}, \bar{t}_{m}+\tau_{m}\right] .
$$

In view of (5.3), for all $m$ sufficiently large,

$$
\text { there exists } t_{m} \in\left[\bar{t}_{m}, \bar{t}_{m}+1\right] \text { such that }\left\|u_{t}\left(t_{m}\right)\right\|_{2}^{2}<2 d_{k} \text {. }
$$

Clearly, up to renaming $\tau_{m}$ into $\left(\tau_{m}-1\right)$ we now have

$$
m^{2} \leq\|u(t)\|_{*}^{2} \leq m^{2}+2 \text { for every } t \in\left[t_{m}, t_{m}+\tau_{m}\right] .
$$

Also, for $m$ large enough, there holds

$$
\int_{\Omega} u\left(t_{m}\right) u_{t}\left(t_{m}\right) d x+\frac{1}{2}\left\|u\left(t_{m}\right)\right\|_{*}^{2} \geq 0 .
$$

Indeed, by (5.10), (5.12), Young, Hölder, and Poincaré inequalities,

$$
\begin{aligned}
\int_{\Omega} u\left(t_{m}\right) u_{t}\left(t_{m}\right) d x+\frac{1}{2}\left\|u\left(t_{m}\right)\right\|_{*}^{2} & \geq \frac{1}{2}\left\|u\left(t_{m}\right)\right\|_{*}^{2}-\left\|u\left(t_{m}\right)\right\|_{2}\left\|u_{t}\left(t_{m}\right)\right\|_{2} \\
& \geq \frac{1}{4}\left\|u\left(t_{m}\right)\right\|_{*}^{2}-c^{\prime}\left\|u_{t}\left(t_{m}\right)\right\|_{2}^{2} \geq \frac{m^{2}}{4}-2 c^{\prime} d_{k} \geq 0
\end{aligned}
$$

for every $m$ large enough. By (5.13) integrating (5.6) on the time interval $\left[t_{m}, t\right]$ for $t$ $\in\left(t_{m}, t_{m}+\tau_{m}\right]$ entails

$$
\int_{\Omega} u(t) u_{t}(t) d x+\frac{1}{2}\|u(t)\|_{*}^{2} \geq \int_{t_{m}}^{t}\left(\frac{(p-1) l-1}{2}\|\nabla u(\tau)\|_{2}^{2}-p E(0)\right) d \tau \geq c^{\prime} \int_{t_{m}}^{t}\|u(\tau)\|_{*}^{2} d \tau
$$

provided $m$ is sufficiently large, where the last inequality follows from (5.7) and the equivalent norm $\|\cdot\|_{*}$ and $H_{0}^{1}(\Omega)$. On the other hand, by Young, Hölder, and Poincaré inequalities, 


$$
\int_{\Omega} u(t) u_{t}(t) d x+\frac{1}{2}\|u(t)\|_{*}^{2} \leq\|u(t)\|_{2}\left\|u_{t}(t)\right\|_{2}+\frac{1}{2}\|u(t)\|_{*}^{2} \leq \frac{1}{2}\left\|u_{t}(t)\right\|_{2}^{2}+c_{2}^{\prime}\|u(t)\|_{*}^{2}
$$

Set

$$
\Gamma_{m}(t)=\int_{t_{m}}^{t}\|u(\tau)\|_{*}^{2} d \tau \quad \text { for every } t \in\left(t_{m}, t_{m}+\tau_{m}\right]
$$

Combining (5.14) with (5.15), we have the following differential inequality

$$
\Gamma_{m}^{\prime}(t) \geq \gamma \Gamma_{m}(t)-c_{3}^{\prime}\left\|u_{t}(t)\right\|_{2}^{2} \quad \text { for every } t \in\left(t_{m}, t_{m}+\tau_{m}\right]
$$

for some $\gamma>0$ and $c_{3}>0$. Hence

$$
\frac{\Gamma_{m}^{\prime}(t)}{\Gamma_{m}(t)} \geq \gamma-c_{3}^{\prime} \frac{\left\|u_{t}(t)\right\|_{2}^{2}}{\Gamma_{m}(t)} \quad \text { for every } t \in\left(t_{m}, t_{m}+\tau_{m}\right]
$$

By (5.12), we have

$$
\Gamma_{m}(t) \geq m^{2}\left(t-t_{m}\right) \geq \frac{m^{2} \tau_{m}}{2} \text { for every } t \in\left[t_{m}+\frac{\tau_{m}}{2}, t_{m}+\tau_{m}\right] .
$$

Then, from (5.16) and (5.17), we obtain

$$
\frac{\Gamma_{m}^{\prime}(t)}{\Gamma_{m}(t)} \geq \gamma-2 c_{3}^{\prime} \frac{\left\|u_{t}(t)\right\|_{2}^{2}}{m^{2} \tau_{m}} \quad \text { for every } t \in\left[t_{m}+\frac{\tau_{m}}{2}, t_{m}+\tau_{m}\right] .
$$

Integrating (5.18) over $\left[t_{m}+\frac{\tau_{m}}{2}, t_{m}+\tau_{m}\right]$ and taking into account (5.3) we find

$$
\log \Gamma_{m}\left(t_{m}+\tau_{m}\right) \geq \log \Gamma_{m}\left(t_{m}+\tau_{m}\right)+\frac{\gamma \tau_{m}}{2}-\frac{2 c^{\prime}{ }_{3} \alpha}{m^{2} \tau_{m}}
$$

where we have set $\alpha=\int_{0}^{\infty}\left\|u_{t}(\tau)\right\|_{2}^{2} d \tau$. Hence, up to enlarging $m$, we may take the exponential and we finally conclude that

$$
\int_{t_{m}}^{t_{m}+\tau_{m}}\|u(\tau)\|_{*}^{2} d \tau=\Gamma_{m}\left(t_{m}+\tau_{m}\right) \geq \frac{1}{2} \Gamma_{m}\left(t_{m}+\frac{\tau_{m}}{2}\right) e^{\gamma \frac{\tau_{m}}{2}} \geq \frac{m^{2} \tau_{m}}{4} e^{\gamma \frac{\tau_{m}}{2}},
$$

where we also used (5.17). On the other hand, by inequality (5.12), it turns out that

$$
\int_{t_{m}}^{t_{m}+\tau_{m}}\|u(\tau)\|_{*}^{2} d \tau \leq\left(m^{2}+2\right) \tau_{m}
$$

which contradicts (5.19) as $\tau_{m} \rightarrow \infty$. Therefor, (5.8) is false and $\{u(t)\}$ is bounded, namely there exists $C$ such that

$$
\left\|u_{t}(t)\right\|_{2}^{2}+\|\nabla u(t)\|_{2}^{2} \leq C, \quad \text { for all } t \geq 0
$$


the Jiangsu Higher Education Committee of China (11KJA110001), the Foundation for Young Talents in College of Anhui Province Grant No. 2011SQRL115, Program sponsored for scientific innovation research of college graduate in Jangsu province No. 181200000649, the pre-research project of Anhui Science and Technology University No. ZRC2012308 and the courses building projects of Anhui Science And Technology University No. ZDKC1121.

\section{Author details}

${ }^{1}$ Jiangsu Provincial Key Laboratory for Numerical Simulation of Large Scale Complex Systems, School of Mathematical Science, Nanjing Normal University, Nanjing 210046, P.R. China ${ }^{2}$ Department of Mathematics, An Hui Science and Technology University, Feng Yang, Anhui 233100, P.R. China

\section{Authors' contributions}

FL and HG carried out all studies in this article. All authors read and approved the final manuscript.

\section{Competing interests}

The authors declare that they have no competing interests.

Received: 12 June 2011 Accepted: 15 February 2012 Published: 15 February 2012

\section{References}

1. Dafermos, M: On abstract Volterra equations with applications to linear viscoelasticiry. J Differ Equ. 7, 554-569 (1970). doi:10.1016/0022-0396(70)90101-4

2. Fabrizio, M, Morro, A: Mathematical Problems in Viscoelasticity. SIAM, Philadephia (1992)

3. Prüss, J: Evolutionary Integral Equations and Applications, Monographs in Mathematics, vol. 87. Birkhaüser, Basel (1993)

4. Renardy, M, Hrusa, WJ, Nohel, JA: Mathematical Problems in Viscoelasticity, Pitman Monographs and Surveys in Pure and Applied Mathematics. Longman, Essex (1987)

5. Appleby, JAD, Fabrizio, M, Lazzari, B, Reynolds, DW: On exponential asymptotic stability in linear viscoelasticity. Math Models Methods Appl Sci. 16, 1677-1694 (2006). doi:10.1142/S0218202506001674

6. Dafermos, M: Asymptotic stabiblity in linear viscoelasticity. Arch Ration Mech Anal. 37, $297-308$ (1970)

7. Dafermos, M: Contraction semigroups and trend to equilibrium in continuum mechanics. Proc IU-TAM/IMU Conference of Applications of Functional Analysis to Mechanics Lecture Notes in Mathematics. pp. 505.Springer, New York (1975)

8. Fabrizio, M, Lazzari, B: On the existence and the asymptotic stability of solutions for linear viscoelastic solids. Arch Ration Mech Anal. 116, 139-152 (1991). doi:10.1007/BF00375589

9. Münoz Rivera, JE, Sobrinho Barbosa, J: Existence and uniform rates of decay for contact problems in viscoelasticity. Appl Anal. 67, 175-199 (1997). doi:10.1080/00036819708840604

10. Mũnoz Rivera, JE, Perla Menzala, G: Decay rates of solutions to a von Kármán system for viscoelastic plates with memory. Quart Appl Math. 57, 181-200 (1999)

11. Fabrizio, M, Polidoro, S: Asymptotic decay for some differential systems with fading memory. Appl Anal. 81, 1245-1264 (2002). doi:10.1080/0003681021000035588

12. Barbu, V, lannelli, M: Controllabiblity of the heat equation with memory. Differ Integr Equ. 13, 1393-1412 (2000)

13. Fabrizio, M, Gentili, G, Reynolds, DW: On rigid linear heat condution with memory. Int J Eng Sci. 36, 765-782 (1998). doi:10.1016/50020-7225(97)00123-7

14. Giorgi, C, Gentili, G: Thermodynamic properties and stability for the heat flux equation with linear memory. Quart Appl Math. 51, 343-362 (1993)

15. Leugering, G: Time optimal boundary controllability of a simple linear viscoelastic fluid. Math Models Methods Appl Sci. 9, 413-430 (1987)

16. Miller, RK: An integrodifferential equation for rigid heat conductors with memory. J Math Anal Appl. 66, 313-332 (1978), doi:10.1016/0022-247X(78)90234-2

17. Cavalcanti, MM, Domingos Cavalcanti, VN, Soriano, JA: Exponential decay for the solution of semilin-ear viscoelastic wave equations with localized damping. Electron J Differ Equ. 44, 1-12 (2002)

18. Cavalcanti, MM, Oquendo, HP: Frictional versus viscoelastic damping in a semilinear wave equation. SIAM J Control Optim. 42(4), 1310-1324 (2003). doi:10.1137/50363012902408010

19. Berrimi, S, Messaoudi, SA: Exponential decay of solutions to a viscoelastic equation with nonlinear localized damping. Electron J Differ Equ. 88, 1-10 (2004)

20. Berrimi, S, Messaoudi, SA: Existence and decay of solutions of a viscoelastic equation with a nonlinear source. Nonlinear Anal. 64, 2314-2331 (2006). doi:10.1016/ina.2005.08.015

21. Messaoudi, SA: Blow up and global existence in a nonlinear viscoelastic wave equation. Math Nachr. 260, 58-66 (2003). doi:10.1002/mana.200310104

22. Georgiev, V, Todorova, G: Existence of a solution of the wave equation with nonlinear damping and source term. J Differ Equ. 109, 295-308 (1994). doi:10.1006/jdeq.1994.1051

23. Messaoudi, SA: Blow up in a nonlinearly damped wave equation. Math Nachr. 231, 1-7 (2001)

24. Messaoudi, SA: Blow up of positive-initial-energy solutions of a nonlinear viscoelastic hyperbolic equation. J Math Anal Appl. 320, 902-915 (2006). doi:10.1016/j.jmaa.2005.07.022

25. Wu, ST: Blow-up of solutions for an integro-differential equation with a nonlinear source. Electron J Differ Equ. 45, 1-9 (2006)

26. Kafini, M, Messaoudi, SA: A blow-up result in a Cauchy viscoelastic problem. Appl Math Lett. 21, 549-553 (2008). doi:10.1016/j.aml.2007.07.004

27. Wang, YJ: A global nonexistence theorem for viscoelastic equations with arbitrarily positive initial energy. Appl Math Lett. 22, 1394-1400 (2009). doi:10.1016/.aml.2009.01.052

28. Payne, L, Sattinger, D: Saddle points and instability on nonlinear hyperbolic equations. Isr Math J. 22, 273-303 (1975). doi:10.1007/BF02761595

29. Sattinger, D: On global solution of nonlinear hyperbolic equations. Arch Ration Mech Anal. 30, 148-172 (1968) 
30. Gazzola, F, Squassina, M: Global solutions and finite time blow up for damped semilinear wave equations. Ann Inst H Poincaré Anal Non Linéaire. 23, 185-207 (2006). doi:10.1016/j.anihpc.2005.02.007

31. Ono, K: On global existence, asymptotic stability and blowing up of solutions for some degenerate non-linear wave equations of Kirchhoff type with a strong dissipation. Math Methods Appl Sci. 20, 151-177 (1997). doi:10.1002/(SICI) 1099-1476(19970125)20:23.0.CO;2-0

32. Tsutsumi, M: On solutions of semilinear differential equations in a Hilbert space. Math J.pn. 17, 173-193 (1972)

33. Haraux, A: Semi-groupes linéires etéquations d' évolution linéaires périodiques. Publication du Laboratoire d'Analyse Numérique, No 78011. Université Pierre et Marie Curie, Paris (1978)

34. Watanabe, K, Yamada, T, Takahashi, W: Reproducing kernels of $H^{m}(a, b)(m=1,2,3)$ and least constants in Sobolevs inequalities. Appl Anal. 82, 809-820 (2003). doi:10.1080/0003681031000152541

35. Levine, HA: Instability and nonexistence of global solutions to nonlinear wave equations of the form. Trans Am Math Soc. 192, 1-21 (1974)

36. Levine, HA: Some additional remarks on the nonexistence of global solutions to nonlinear wave equations. SIAM J Math Anal. 5, 138-146 (1974). doi:10.1137/0505015

37. Ikehata, R: Some remarks on the wave equations with nonlinear damping and source terms. Nonlinear Anal. 27, 1165-1175 (1996). doi:10.1016/0362-546X(95)00119-G

38. Gazzola, F, Weth, T: Finite time blow-up and global solutions for semilinear parabolic equations with initial data at high energy level. Diff Integr Equ. 18(9), 961-990 (2005)

doi:10.1186/1029-242X-2012-33

Cite this article as: Liang and Gao: Global existence and blow-up of solutions for a nonlinear wave equation with memory. Journal of Inequalities and Applications 2012 2012:33.

\section{Submit your manuscript to a SpringerOpen ${ }^{\odot}$} journal and benefit from:

Convenient online submission

Rigorous peer review

- Immediate publication on acceptance

- Open access: articles freely available online

- High visibility within the field

- Retaining the copyright to your article

Submit your next manuscript at $\boldsymbol{\wedge}$ springeropen.com 